\title{
In vivo direct imaging of neuronal activity at high temporo-spatial
}

\section{resolution}

Phan Tan Toi ${ }^{1,2,3 \dagger}$, Hyun Jae Jang ${ }^{4 \dagger}$, Kyeongseon Min ${ }^{5}$, Sung-Phil Kim ${ }^{6}$, Seung-Kyun Lee ${ }^{1,2,3}$, Jongho Lee ${ }^{5}$, Jeehyun Kwag ${ }^{4 *}$, Jang-Yeon Park ${ }^{1,2,3 *}$

${ }^{1}$ Department of Biomedical Engineering, Sungkyunkwan University; Suwon 16419, Republic of Korea.

${ }^{2}$ Department of Intelligent Precision Healthcare Convergence, Sungkyunkwan University; Suwon 16419, Republic of Korea.

${ }^{3}$ Center for Neuroscience Imaging Research, Institute for Basic Science; Suwon 16419, Republic of Korea.

${ }^{4}$ Department of Brain and Cognitive Engineering, Korea University; Seoul 02841, Republic of Korea.

$15{ }^{5}$ Department of Electrical and Computer Engineering, Seoul National University; Seoul 08826, Republic of Korea.

${ }^{6}$ Department of Biomedical Engineering, Ulsan National Institute of Science and Technology; Ulsan 44919, Republic of Korea.

$\dagger$ These authors contributed equally to this work.

* Corresponding authors. Email: jyparu@skku.edu (J.Y.P.); jkwag@korea.ac.kr (J.K.) 


\begin{abstract}
There has been a longstanding demand for noninvasive neuroimaging methods capable of detecting neuronal activity at both high temporal and spatial resolution. Here, we propose a novel method that enables Direct Imaging of Neuronal Activity for functional MRI (termed DIANAfMRI) that can dynamically image spiking activity in milliseconds precision, while retaining the original benefit of high spatial resolution of MRI. DIANA-fMRI was demonstrated through in vivo mice brain imaging at 9.4 $\mathrm{T}$ applying electrical whisker-pad stimulation, directly imaging the spiking activity as well as capturing its sequential propagation along the thalamocortical pathway, as further confirmed through in vivo spike recording and optogenetics. DIANA-fMRI will open up new avenues in brain science by providing a deeper understanding of the brain's functional organization including neural networks.
\end{abstract}




\section{Introduction}

Advanced noninvasive neuroimaging methods provide valuable information on the brain's functional organization, but they have obvious pros and cons in terms of temporal and spatial resolution. Functional magnetic resonance imaging (fMRI) using blood-oxygenation-leveldependent (BOLD) effect provides good spatial resolution in the order of millimeters, but has a poor temporal resolution in the order of seconds due to slow hemodynamic responses to neuronal activation (1), providing only indirect information on neuronal activity through neurovascular coupling. In contrast, electroencephalography (EEG) and magnetoencephalography (MEG) provide excellent temporal resolution in the millisecond range, but spatial information is limited to centimeter scales (2). Thus, while harnessing the high spatial resolution of MRI, enhancement of MRI-based temporal resolution up to that of EEG or MEG that can directly measure neuronal activity in the order of milliseconds is imperative to advance the understanding of in vivo brain, especially to elucidate the causal link between the in vivo neuronal activities and brain function.

Over the past decades, many attempts have been made in exploring the possibility of using MRI to directly image neuronal activity (3). Most of the studies were based on the neuronal current models, where neuronal currents flowing along the axon produce an ultraweak circumferential magnetic field on the order of nanotesla $(\mathrm{nT})(4,5)$, thereby locally changing the phase and magnitude of MR signals. Phantom studies demonstrated small phase shifts $\left(<1^{\circ}\right)$ induced by magnetic field changes $\leq 1 \mathrm{nT}$ when injecting electrical currents through wires in a gel phantom $(4,6-8)$. The in vitro studies using hemoglobin-free biological objects such as rat neuron cells $(9)$, snail ganglia $(10,11)$, isolated brain of turtle $(8,12)$, and octopus $(13)$ also reported changes in the magnitude of MR signals (e.g., $0.01-5.5 \%$ ) induced by the applied stimulus. More interestingly, some in vivo studies have argued that they succeeded in directly detecting the human brain 
activation in vivo in response to event-related tasks (14-19), but they have not been replicated in later attempts $(20-24)$.

Despite many efforts to use MRI to directly detect neuronal activity, no firm consensus has been reached on its feasibility yet, especially in in vivo studies. Here, we propose a novel method that enables Direct Imaging of Neuronal Activity for functional MRI (termed DIANA-fMRI) by increasing the temporal resolution up to the temporal precision of neuronal activity in milliseconds, while retaining the original benefit of high spatial resolution of MRI.

\section{Results}

\section{Magnetic resonance imaging of neuronal activity at milliseconds temporal resolution}

To implement high temporal resolution in milliseconds scale, we combined the line scanning method (25) and fast low angle shot (FLASH) gradient-echo imaging, where a single line of $k$ space was repeatedly acquired during each interstimulus interval, and different $k$-space lines were acquired in different periods. In this scheme, each stimulation period adds one line of the $k$-space to all the time-series images within the period (Fig. 1A) $(26,27)$, and the repetition time (TR) of the FLASH sequence exactly determines the temporal resolution of the dynamic imaging in DIANA-fMRI. Under this paradigm, electrical stimulation was repeatedly applied at $200 \mathrm{~ms}$ interstimulus intervals, determined by $5 \mathrm{~ms}$ TR multiplied by 40 frames in the time series. DIANAfMRI showed similar performance to regular two-dimensional FLASH imaging in terms of signalto-noise ratio (SNR) and temporal SNR (tSNR) (fig. S1). To test whether DIANA-fMRI can directly detect neuronal activity, we delivered electrical stimulation to the left whisker-pad (strength, $0.5 \mathrm{~mA}$; duration, $0.5 \mathrm{~ms}$; frequency, $5 \mathrm{~Hz}$ ) of anesthetized mice placed inside the $9.4 \mathrm{~T}$ scanner and imaged a single $1 \mathrm{~mm}$ coronal brain slice which included the right barrel field of 
primary somatosensory cortex $(\mathrm{S} 1 \mathrm{BF})$ that is contralateral to the left whisker pad (Fig. 1B). In response to the electrical whisker-pad stimulation, a statistically significant increase in the DIANA signal was observed from the contralateral S1BF compared to the pre-stimulus signal $(0.157 \pm$ $0.015 \%, p<0.001,5$ mice) (Fig. 1, C to E; fig. S2), whereas there were no significant changes in the unstimulated control mice, postmortem mice (Fig. 1, C to E; fig. S2) nor in sham experiments performed using an agar phantom (fig. S3). Most interestingly, the peak DIANA signal occurred with a latency of $24.00 \pm 2.92 \mathrm{~ms}$ after the whisker-pad stimulation onset (Fig. 1, C, D and F), indicating that DIANA can detect whisker-pad stimulation-evoked responses by achieving high temporal resolution in milliseconds range. To find the neural correlates of DIANA-fMRI in vivo, the same whisker-pad stimulation paradigm as in Fig. 1B was repeated but now in mice implanted with a 32-channel silicon probe in the S1BF (Fig. 1G) to record the local field potential (LFP) and single-unit spike activities (Fig. 1H), from which their latencies were analyzed (Fig. 1, I to K). The peak of whisker-pad stimulation-evoked LFP had a latency of $39.48 \pm 1.84 \mathrm{~ms}$ (12 mice), which was significantly slower than the latency of DIANA response ( $p<0.001$, Fig. 1 , I and K). However, the peak spike firing rates of whisker-pad stimulation-responsive single units had a latency of $26.44 \pm 1.24 \mathrm{~ms}$ (27 units from 10 mice) in the post-stimulus time histogram (PSTH) (Fig. 1J), which, surprisingly, was similar to the DIANA response latency as their latencies were not statistically different $(p>0.05$, Fig. $1 \mathrm{~K})$. Additionally, other temporal spike characteristics such as time-to-first spike latency as well as median and mode of whisker-pad stimulationresponsive spike timings were also similar to the DIANA response latency (fig. S4). DIANA response amplitudes and spike firing rates both increased with increasing strength of electrical whisker-pad stimulation with little change in the latencies of their peaks (fig. S5). Together, these 
results show for the first time that DIANA-fMRI can measure transient neuronal activity with milliseconds temporal resolution that is comparable to the single-unit spike recordings in vivo.

\section{Temporo-spatial imaging of neuronal activity propagation}

Since somatosensory stimulus-evoked spikes propagate to S1BF via thalamus in the thalamocortical pathway $(28,29)$, we next explored if the high temporo-spatial resolution $(5 \mathrm{~ms}$, $0.22 \mathrm{~mm}$ ) of DIANA-fMRI can also capture the propagation of spikes. When a $1 \mathrm{~mm}$ coronal brain slice that included both the thalamus and S1BF was imaged applying electrical whisker-pad stimulation (Fig. 2A), while conventional BOLD-fMRI showed concomitant activation of thalamus, contra- and ipsilateral S1BF (Fig. 2B and fig. S6), DIANA-fMRI showed statistically significant DIANA response which was sequentially activated in the order of thalamus $(0.157 \pm$ $0.011 \%)$ and, almost simultaneously, contralateral S1BF $(0.161 \pm 0.009 \%)$ and ipsilateral S1BF $(0.047 \pm 0.014 \%)$ (Fig. $2, \mathrm{C}$ and D; fig. S7, A and B) with latencies of $11.50 \pm 0.76 \mathrm{~ms}, 25.00 \pm$ $1.49 \mathrm{~ms}$, and $25.00 \pm 3.16 \mathrm{~ms}$ (Fig. 2E and fig. S7C) following stimulation onset, respectively. Further cross-correlation analysis of the time series confirmed that thalamic responses precede contra- and ipsilateral S1BF by $10-15 \mathrm{~ms}$ (fig. S8). To see if the temporo-spatial propagation of DIANA-fMRI matches that of spikes in the thalamocortical pathway, we performed simultaneous single-unit recordings in the thalamus and S1BF using two silicon probes with electrical whiskerpad stimulation (Fig. 2, F to I). Consistent with DIANA responses, latencies of peak spike firing rates of the stimulation-responsive single units occurred in the order of the thalamus $(9.52 \pm 0.90$ ms, 23 units from 10 mice), contralateral S1BF (24.52 $\pm 1.43 \mathrm{~ms}, 23$ units from 5 mice) (Fig. 2, I and J) and ipsilateral S1BF (30.00 $\pm 1.73 \mathrm{~ms}, 3$ units from 1 mouse) (fig. S9). Such sequential propagation of spikes in the thalamus and the S1BF was statistically similar to those observed from 
DIANA responses (Fig. 2E and fig. S10). LFP also showed sequential propagation in the thalamus $(31.78 \pm 3.67 \mathrm{~ms})$, contralateral S1BF $(42.69 \pm 3.04 \mathrm{~ms})$, and ipsilateral S1BF $(57.67 \pm 5.33 \mathrm{~ms})$, but they were significantly slower than the DIANA responses (fig. S10). These results demonstrate that DIANA-fMRI can capture spike propagation across functionally-defined thalamocortical pathway with high temporo-spatial resolution.

\section{Optogenetic DIANA-fMRI experiment}

Although the temporal characteristics of DIANA responses and electrophysiologically recorded spikes in vivo are statistically similar, these measurements are somewhat limited since spike activity in vivo, we employed an optogenetic fMRI scheme. DIANA responses were measured during optogenetic activation of Channelrhodopsin2 (ChR2)-expressing excitatory neurons in the S1BF with $473 \mathrm{~nm}$ blue light delivered through chronically implanted fiber-optic cannula (Fig. 3A). Immunostaining showed that stereotaxically injected AAV5-CamKIIahChR2(ET/TC)-mCherry (30) into S1BF expressed ChR2-mCherry in excitatory neurons across all cortical layers (Fig. 3B). During blue light stimulation (intensity, $50 \mathrm{~mW} / \mathrm{mm}^{2}$; duration, 20 ms), DIANA responses were acquired as a time series of 50 images every $5 \mathrm{~ms}$ from a $1 \mathrm{~mm}$ coronal brain slice containing both the thalamus and S1BF (Fig. 3C). We found statistically significant DIANA signal change in the S1BF $(0.200 \pm 0.070 \%)$ with response latencies of 13.75 $\pm 4.27 \mathrm{~ms}$, which was followed by significant DIANA signal change in the thalamus $(0.217 \pm$ $0.081 \%) 37.50 \pm 8.78 \mathrm{~ms}$ after stimulation onset (Fig. 3, D and E), directly imaging optogenetic blue light stimulation-evoked neuronal activities as well as capturing the feedback spike propagation in the cortico-thalamic pathway $(29,31,32)$. Indeed, when we recorded blue light 
stimulation-evoked single-unit activities from the S1BF and the thalamus of the same mice that were used in optogenetic DIANA-fMRI experiment (Fig. 3F), peak spike firing rate of neurons in S1BF occurred $9.06 \pm 1.59 \mathrm{~ms}$ after stimulation onset (34 units from 8 mice), followed by thalamic peak spike firing rate occurring at $21.86 \pm 2.76 \mathrm{~ms}$ ( 7 units from 4 mice) (Fig. 3, G to I), showing feedback spike propagation in the cortico-thalamic pathway. In addition, both DIANA responses and peak spike firing rates increased with increasing blue light stimulation duration (fig. S11), indicating similarities of DIANA responses and single-unit activities. Although feedback propagation of LFP in the cortico-thalamic pathway was also observed (S1BF, $22.34 \pm 0.87 \mathrm{~ms}$; thalamus, $77.55 \pm 4.61 \mathrm{~ms}$ ), peak LFP latencies were significantly slower than those of optogenetic DIANA responses (fig. S12). Together, optogenetic DIANA and corresponding in vivo single-unit recordings further confirm that the DIANA response is indeed consequences of direct detection of the spike activity in vivo.

\section{$D I A N A$ response as non-BOLD effect}

Despite convincing evidence presented here for DIANA's ability to directly detect neuronal activity, it is possible that DIANA response might involve hemodynamic responses such as the BOLD effect. To dissect the BOLD effect from the DIANA response, BOLD-fMRI experiments were performed under two conditions: one condition as a default with extra oxygen-to-air ratio of 1:4 (oxygen:air condition) and the other condition with air only (Fig. 4A). The BOLD responses in the thalamus and S1BF following electrical whisker-pad stimulation in the air-only condition were significantly reduced compared to the oxygen:air condition (thalamus: $0.589 \pm 0.045 \%$ to $0.251 \pm 0.081 \%$; contralateral S1BF: $1.128 \pm 0.099 \%$ to $0.786 \pm 0.127 \%$, Fig. 4 , B and C), consistent with BOLD activation's dependence on oxygen supply $(33,34)$. In contrast, there was 
little change in the DIANA responses between air-only and oxygen:air conditions (thalamus: $0.188 \pm 0.013$ to $0.189 \pm 0.009 \%$; contralateral S1BF: $0.165 \pm 0.009 \%$ to $0.168 \pm 0.011 \%$, Fig. 4 , $\mathrm{D}$ and $\mathrm{E})$. These results show that the hemodynamic BOLD response is not involved in the DIANA contrast mechanism.

Then what could be the possible signal source of DIANA response? Electromagnetic effects based on popular neuronal current models cannot be a candidate since signal loss caused by the phase cancellation of proton spins under the neuronal current-induced magnetic field change has a negative value $(3,4,9,15)$ while DIANA response has a positive signal change in the main lobe.

Instead, because changes in neuronal membrane potential can induce reorientation of the membrane interfacial water (35), DIANA response may arise from the changes in MR relaxation times $\left(\mathrm{T}_{1}, \mathrm{~T}_{2}\right)$ during neuronal activity, which is closely related to the amount of water molecules in the hydration layer on the membrane surface (36). To test our hypothesis, we performed phantom experiments using $T$ cells to measure $T_{1}$ and $T_{2}$ relaxation times while $T$ cells membrane potentials were manipulated by different extracellular $\mathrm{K}^{+}$concentrations $\left(\left[\mathrm{K}^{+}\right]\right)(37)(4.2$ to 141.0 mM, Fig. 4F). We used T cells as phantom since, unlike neurons, $\mathrm{T}$ cells are homogeneous and immortal that can survive without oxygen supply in vitro in the scanner. $\mathrm{T}_{1}$ and $\mathrm{T}_{2}$ relaxation time maps (Fig. 4G) and values showed a strong positive correlation with $\left[\mathrm{K}^{+}\right]\left(\mathrm{T}_{1}\right.$ slope, $0.956 \mathrm{~ms} / \mathrm{mM}$, $\mathrm{R}^{2}=0.884 ; \mathrm{T}_{2}$ slope, $0.106 \mathrm{~ms} / \mathrm{mM}, \mathrm{R}^{2}=0.889$, Fig. $\left.4 \mathrm{H}\right)$. Thus, it is possible that the release of hydrated water into the free water around the cell membrane during membrane depolarization may account for $T_{1}$ and $T_{2}$ changes. Based on these measurements of $T_{1}$ and $T_{2}$ changes, Bloch simulation was performed to estimate DIANA signal changes and showed a positive signal change of $\sim 0.13 \%$ in the main lobe (fig. S13), which is in good agreement with the experimental results (Figs. 1 to 3) and supports our hypothesis about the DIANA contrast mechanism. 


\section{Discussion}

Together, our results demonstrate that DIANA-fMRI indeed enables direct mapping of spike activity in vivo with high temporal $(5 \mathrm{~ms})$ and spatial $(0.22 \mathrm{~mm})$ resolution, as confirmed through in vivo electrophysiology combined with optogenetics. Such high temporal resolution of DIANA-fMRI even allowed the detection of the sequential propagation of neuronal activity through functionally-defined neural networks in the thalamocortical as well as cortico-thalamic pathways.

However, DIANA-fMRI has some limitations at this stage. Because its milliseconds temporal resolution is achieved based on line-scan data acquisition, DIANA-fMRI data is only generated in event-related responses to repetitive stimulation applications and, thus, it is challenging to investigate brain function in the resting state, or with a single stimulus. Moreover, DIANA-fMRI assumes that event-related responses in the brain are consistent across all the responses without trial-by-trial variability, which can somewhat undermine the reliability of DIANA-fMRI even with a modest change in neural spiking with each stimulation (38). Further investigation is also needed to fully elucidate the contrast mechanism of DIANA-fMRI.

There are interesting topics that can immediately be explored with DIANA-fMRI. One is to investigate whether DIANA-fMRI can noninvasively reveal rapid neural network dynamics in functionally-connected multiple, distant brain regions with a time scale of neural spike $(3,39)$. Furthermore, while we presented the DIANA-fMRI data detecting only fast-phasic neuronal activity here, by adjusting the inter-stimulation period long enough to measure the neuronal activity of interest, DIANA-fMRI could be used to measure diverse temporal patterns of neuronal activity on various time scales such as sustained tonic neuronal activity over a longer period of 
time. Another is to test its feasibility for human fMRI and translate DIANA-fMRI into a clinical human system. Approximate predictions only taking into account neuronal density, magnetic field strength, and typical voxel size in the animal and human systems suggest that DIANA-fMRI is likely to work in human studies as well (Supplementary Text). More complicated feedforward and feedback responses are expected to be observed in human brain networks (40), which could make DIANA response more challenging but even more interesting.

Overall, we expect DIANA-fMRI to open up new avenues in neuroimaging for a more accurate and deeper understanding of the brain's functional organization, especially elucidating the causal relationship between temporal dynamics of neural networks and their function through the convergence of high temporal and spatial resolution. 


\section{References}

1. S. Ogawa, T. M. Lee, A. R. Kay, D. W. Tank, Brain magnetic resonance imaging with contrast dependent on blood oxygenation. PNAS. 87, 9868-9872 (1990), doi:10.1073/pnas.87.24.9868, pmid:2124706.

2. D. Cohen, Magnetoencephalography: Evidence of Magnetic Fields Produced by AlphaRhythm Currents. Science. 161, 784-786 (1968), doi:10.1126/science.161.3843.784, pmid:5663803.

3. P. A. Bandettini, N. Petridou, J. Bodurka, Direct detection of neuronal activity with MRI: Fantasy, possibility, or reality? Appl. Magn. Reson. 29, 65-88 (2005), doi:10.1007/BF03166956.

4. J. Bodurka, P. A. Bandettini, Toward direct mapping of neuronal activity: MRI detection of ultraweak, transient magnetic field changes. Magnetic Resonance in Medicine. 47, 1052-1058 (2002), doi:10.1002/mrm.10159, pmid:12111950.

5. D. Konn, P. Gowland, R. Bowtell, MRI detection of weak magnetic fields due to an extended current dipole in a conducting sphere: A model for direct detection of neuronal currents in the brain. Magnetic Resonance in Medicine. 50, 40-49 (2003), doi:10.1002/mrm.10494, pmid: 12815677.

6. M. Singh, Sensitivity of MR phase shift to detect evoked neuromagnetic fields inside the head. IEEE Transactions on Nuclear Science. 41, 349-351 (1994), doi:10.1109/23.281521.

7. J. Bodurka, A. Jesmanowicz, J. S. Hyde, H. Xu, L. Estkowski, S.-J. Li, Current-Induced Magnetic Resonance Phase Imaging. Journal of Magnetic Resonance. 137, 265-271 (1999), doi:10.1006/jmre.1998.1680, pmid:10053158. 
8. P. Sundaram, A. Nummenmaa, W. Wells, D. Orbach, D. Orringer, R. Mulkern, Y. Okada, Direct neural current imaging in an intact cerebellum with magnetic resonance imaging. NeuroImage. 132, 477-490 (2016), doi:10.1016/j.neuroimage.2016.01.059, pmid:26899788.

9. N. Petridou, D. Plenz, A. C. Silva, M. Loew, J. Bodurka, P. A. Bandettini, Direct magnetic resonance detection of neuronal electrical activity. PNAS. 103, 16015-16020 (2006), doi:10.1073/pnas.0603219103, pmid:17038505.

10. T. S. Park, S. Y. Lee, J.-H. Park, S. Y. Lee, Effect of nerve cell currents on MRI images in snail ganglia. NeuroReport. 15, 2783-2786 (2004), pmid:15597054.

11. T. S. Park, S. Y. Lee, J.-H. Park, M. H. Cho, S. Y. Lee, Observation of the fast response of a magnetic resonance signal to neuronal activity: a snail ganglia study. Physiol. Meas. 27, 181 (2006), doi:10.1088/0967-3334/27/2/008, pmid:16400204.

12. Q. Luo, H. Lu, H. Lu, D. Senseman, K. Worsley, Y. Yang, J.-H. Gao, Physiologically evoked neuronal current MRI in a bloodless turtle brain: Detectable or not? NeuroImage. 47, 12681276 (2009), doi:10.1016/j.neuroimage.2009.06.017, pmid:19539040.

13. X. Jiang, H. Lu, S. Shigeno, L.-H. Tan, Y. Yang, C. W. Ragsdale, J.-H. Gao, Octopus visual system: A functional MRI model for detecting neuronal electric currents without a bloodoxygen-level-dependent confound. Magnetic Resonance in Medicine. 72, 1311-1319 (2014), doi:10.1002/mrm.25051, pmid:24301336.

14. M. Joy, G. Scott, M. Henkelman, In vivo detection of applied electric currents by magnetic resonance imaging. Magnetic Resonance Imaging. 7, 89-94 (1989), doi:10.1016/0730725X(89)90328-7, pmid:2918822. 
15. J. Xiong, P. T. Fox, J.-H. Gao, Directly mapping magnetic field effects of neuronal activity by magnetic resonance imaging. Human Brain Mapping. 20, 41-49 (2003), doi:10.1002/hbm.10124, pmid:12953305.

16. L. S. Chow, G. G. Cook, E. Whitby, M. N. J. Paley, Investigating direct detection of axon firing in the adult human optic nerve using MRI. NeuroImage. 30, 835-846 (2006), doi:10.1016/j.neuroimage.2005.10.024, pmid:16376108.

17. L. S. Chow, G. G. Cook, E. Whitby, M. N. J. Paley, Investigation of MR signal modulation due to magnetic fields from neuronal currents in the adult human optic nerve and visual cortex. Magnetic Resonance Imaging. 24, 681-691 (2006), doi:10.1016/j.mri.2006.01.005, pmid:16824962.

18. L. S. Chow, G. G. Cook, E. Whitby, M. N. J. Paley, Investigation of axonal magnetic fields in the human corpus callosum using visual stimulation based on MR signal modulation. Journal of Magnetic Resonance Imaging. 26, 265-273 (2007), doi:10.1002/jmri.21025, pmid:17654726.

19. L. S. Chow, A. Dagens, Y. Fu, G. G. Cook, M. N. J. Paley, Comparison of BOLD and directMR neuronal detection (DND) in the human visual cortex at 3T. Magnetic Resonance in Medicine. 60, 1147-1154 (2008), doi:10.1002/mrm.21753, pmid:18956466.

20. R. Chu, J. A. de Zwart, P. van Gelderen, M. Fukunaga, P. Kellman, T. Holroyd, J. H. Duyn, Hunting for neuronal currents: absence of rapid MRI signal changes during visual-evoked response. NeuroImage. 23, 1059-1067 (2004), doi:10.1016/j.neuroimage.2004.07.003, pmid:15528106. 
21. L. M. Parkes, F. P. de Lange, P. Fries, I. Toni, D. G. Norris, Inability to directly detect magnetic field changes associated with neuronal activity. Magnetic Resonance in Medicine. 57, 411416 (2007), doi:10.1002/mrm.21129, pmid:17260380.

22. L. Tang, M. J. Avison, J. C. Gatenby, J. C. Gore, Failure to direct detect magnetic field dephasing corresponding to ERP generation. Magnetic Resonance Imaging. 26, 484-489 (2008), doi:10.1016/j.mri.2007.09.003, pmid:18180125.

23. J. Huang, Detecting neuronal currents with MRI: A human study. Magnetic Resonance in Medicine. 71, 756-762 (2014), doi:10.1002/mrm.24720, pmid:23475847.

24. J. Huang, D. C. Zhu, Exploring human brain neuronal currents with phase MRI. International Journal of Imaging Systems and Technology. 25, 172-178 (2015), doi:10.1002/ima.22134.

25. X. Yu, C. Qian, D. Chen, S. J. Dodd, A. P. Koretsky, Deciphering laminar-specific neural inputs with line-scanning fMRI. Nat Methods. 11, 55-58 (2014), doi:10.1038/nmeth.2730, pmid:24240320.

26. J. Lee, S.-K. Lee, J.-Y. Park, in Proceedings of Scientific Meeting of the International Society for Magnetic Resonance in Medicine (Paris, France, 2018), vol. 26, p. 0703.

27. P. T. Toi, H. Lee, J. Lee, S.-K. Lee, J.-Y. Park, in Proceedings of Scientific Meeting of the International Society for Magnetic Resonance in Medicine (Montreal, QC, Canada, 2019; ISMRM), vol. 27, p. 2991.

28. E. Ahissar, R. Sosnik, S. Haidarliu, Transformation from temporal to rate coding in a somatosensory thalamocortical pathway. Nature. 406, 302-306 (2000), doi:10.1038/35018568, pmid:10917531. 
29. M. Deschênes, P. Veinante, Z.-W. Zhang, The organization of corticothalamic projections: reciprocity versus parity. Brain Research Reviews. 28, 286-308 (1998), doi:10.1016/S01650173(98)00017-4, pmid:9858751.

30. A. Berndt, P. Schoenenberger, J. Mattis, K. M. Tye, K. Deisseroth, P. Hegemann, T. G. Oertner, High-efficiency channelrhodopsins for fast neuronal stimulation at low light levels. PNAS. 108, 7595-7600 (2011), doi:10.1073/pnas.1017210108, pmid:21504945.

31. J. H. Lee, R. Durand, V. Gradinaru, F. Zhang, I. Goshen, D.-S. Kim, L. E. Fenno, C. Ramakrishnan, K. Deisseroth, Global and local fMRI signals driven by neurons defined optogenetically by type and wiring. Nature. 465, 788-792 (2010), doi:10.1038/nature09108, pmid:20473285.

32. M. A. Kirchgessner, A. D. Franklin, E. M. Callaway, Context-dependent and dynamic functional influence of corticothalamic pathways to first- and higher-order visual thalamus. PNAS. 117, 13066-13077 (2020), doi:10.1073/pnas.2002080117, pmid:32461374.

33. K. M. Sicard, T. Q. Duong, Effects of hypoxia, hyperoxia, and hypercapnia on baseline and stimulus-evoked BOLD, CBF, and CMRO2 in spontaneously breathing animals. NeuroImage. 25, 850-858 (2005), doi:10.1016/j.neuroimage.2004.12.010, pmid:15808985.

34. T. Q. Duong, Cerebral blood flow and BOLD fMRI responses to hypoxia in awake and anesthetized rats. Brain Research. 1135, 186-194 (2007), doi:10.1016/j.brainres.2006.11.097, pmid:17198686.

35. M. E. P. Didier, O. B. Tarun, P. Jourdain, P. Magistretti, S. Roke, Membrane water for probing neuronal membrane potentials and ionic fluxes at the single cell level. Nature Communications. 9, 5287 (2018), doi:10.1038/s41467-018-07713-w, pmid:30538243. 
36. K. Min, P. T. Toi, S. Chung, J. Lee, S.-K. Lee, J.-Y. Park, in Proceedings of Scientific Meeting of the International Society for Magnetic Resonance in Medicine (Virtual Conference, 2020; ISMRM), vol. 28, p. 0173.

37. F. Mello de Queiroz, C. G. Ponte, A. Bonomo, R. Vianna-Jorge, G. Suarez-Kurtz, Study of membrane potential in $\mathrm{T}$ lymphocytes subpopulations using flow cytometry. $B M C$ Immunology. 9, 63 (2008), doi:10.1186/1471-2172-9-63, pmid:18980671.

38. E. Kheradpezhouh, M. Adibi, E. Arabzadeh, Response dynamics of rat barrel cortex neurons to repeated sensory stimulation. Scientific Reports. 7, 11445 (2017), doi:10.1038/s41598-01711477-6, pmid:28904406.

39. I. Magrans de Abril, J. Yoshimoto, K. Doya, Connectivity inference from neural recording data: Challenges, mathematical bases and research directions. Neural Networks. 102, 120-137 (2018), doi:10.1016/j.neunet.2018.02.016, pmid:29571122.

40. O. Sporns, D. R. Chialvo, M. Kaiser, C. C. Hilgetag, Organization, development and function of complex brain networks. Trends in Cognitive Sciences. 8, 418-425 (2004), doi:10.1016/j.tics.2004.07.008, pmid:15350243.

41. H.-J. Shim, W. B. Jung, F. Schlegel, J. Lee, S. Kim, J. Lee, S.-G. Kim, Mouse fMRI under ketamine and xylazine anesthesia: Robust contralateral somatosensory cortex activation in response to forepaw stimulation. NeuroImage. 177, 30-44 (2018), doi:10.1016/j.neuroimage.2018.04.062, pmid:29730495.

42. E. S. Boyden, F. Zhang, E. Bamberg, G. Nagel, K. Deisseroth, Millisecond-timescale, genetically targeted optical control of neural activity. Nature Neuroscience. 8, 1263-1268 (2005), doi:10.1038/nn1525, pmid:16116447.

43. F. Bloch, Nuclear Induction. Phys. Rev. 70, 460-474 (1946), doi:10.1103/PhysRev.70.460. 
44. E. S. Lein, M. J. Hawrylycz, N. Ao, M. Ayres, A. Bensinger, A. Bernard, A. F. Boe, M. S. Boguski, K. S. Brockway, E. J. Byrnes, L. Chen, L. Chen, T.-M. Chen, M. C. Chin, J. Chong, B. E. Crook, A. Czaplinska, C. N. Dang, S. Datta, N. R. Dee, A. L. Desaki, T. Desta, E. Diep, T. A. Dolbeare, M. J. Donelan, H.-W. Dong, J. G. Dougherty, B. J. Duncan, A. J. Ebbert, G. Eichele, L. K. Estin, C. Faber, B. A. Facer, R. Fields, S. R. Fischer, T. P. Fliss, C. Frensley, S. N. Gates, K. J. Glattfelder, K. R. Halverson, M. R. Hart, J. G. Hohmann, M. P. Howell, D. P. Jeung, R. A. Johnson, P. T. Karr, R. Kawal, J. M. Kidney, R. H. Knapik, C. L. Kuan, J. H. Lake, A. R. Laramee, K. D. Larsen, C. Lau, T. A. Lemon, A. J. Liang, Y. Liu, L. T. Luong, J. Michaels, J. J. Morgan, R. J. Morgan, M. T. Mortrud, N. F. Mosqueda, L. L. Ng, R. Ng, G. J. Riley, H. R. Rockett, S. A. Rowland, J. J. Royall, M. J. Ruiz, N. R. Sarno, K. Schaffnit, N. V. Shapovalova, T. Sivisay, C. R. Slaughterbeck, S. C. Smith, K. A. Smith, B. I. Smith, A. J. Sodt, N. N. Stewart, K.-R. Stumpf, S. M. Sunkin, M. Sutram, A. Tam, C. D. Teemer, C. Thaller, C. L. Thompson, L. R. Varnam, A. Visel, R. M. Whitlock, P. E. Wohnoutka, C. K. Wolkey, V. Y. Wong, M. Wood, M. B. Yaylaoglu, R. C. Young, B. L. Youngstrom, X. F. Yuan, B. Zhang, T. A. Zwingman, A. R. Jones, Genome-wide atlas of gene expression in the adult mouse brain. Nature. 445, 168-176 (2007), doi:10.1038/nature05453, pmid:17151600.

45. R. W. Cox, AFNI: Software for Analysis and Visualization of Functional Magnetic Resonance Neuroimages. Computers and Biomedical Research. 29, 162-173 (1996), doi:10.1006/cbmr.1996.0014, pmid:8812068.

46. S. M. Smith, M. Jenkinson, M. W. Woolrich, C. F. Beckmann, T. E. J. Behrens, H. JohansenBerg, P. R. Bannister, M. De Luca, I. Drobnjak, D. E. Flitney, R. K. Niazy, J. Saunders, J. Vickers, Y. Zhang, N. De Stefano, J. M. Brady, P. M. Matthews, Advances in functional and 
structural MR image analysis and implementation as FSL. NeuroImage. 23, S208-S219 (2004), doi:10.1016/j.neuroimage.2004.07.051, pmid:15501092.

47. B. B. Avants, N. J. Tustison, G. Song, P. A. Cook, A. Klein, J. C. Gee, A reproducible evaluation of ANTs similarity metric performance in brain image registration. NeuroImage. 54, 2033-2044 (2011), doi:10.1016/j.neuroimage.2010.09.025, pmid:20851191.

48. M. Pachitariu, N. A. Steinmetz, S. N. Kadir, M. Carandini, K. D. Harris, in Advances in Neural Information Processing Systems 29, D. D. Lee, M. Sugiyama, U. V. Luxburg, I. Guyon, R. Garnett, Eds. (Curran Associates, Inc., 2016; http://papers.nips.cc/paper/6326-fast-andaccurate-spike-sorting-of-high-channel-count-probes-with-kilosort.pdf), pp. 4448-4456. parvalbumin and somatostatin interneurons in gating the synchronization of spike times in the neocortex. Sci Adv. 6, eaay5333 (2020), doi:10.1126/sciadv.aay5333, pmid:32426459.

50. A. L. Barth, J. F. A. Poulet, Experimental evidence for sparse firing in the neocortex. Trends in Neurosciences. 35, 345-355 (2012), doi:10.1016/j.tins.2012.03.008, pmid:22579264. Mouse Olfactory Bulb. Cell Rep. 21, 919-925 (2017), doi:10.1016/j.celrep.2017.09.094, pmid:29069599.

52. D. C. V. Essen, C. J. Donahue, T. S. Coalson, H. Kennedy, T. Hayashi, M. F. Glasser, Cerebral cortical folding, parcellation, and connectivity in humans, nonhuman primates, and mice. PNAS. 116, 26173-26180 (2019), doi:10.1073/pnas.1902299116, pmid:31871175.

Acknowledgments: We thank Prof. Seong-Gi Kim's group and Dr. Geunho Im for helping setting up mouse BOLD-fMRI experiments at the beginning of this study. We also thank Drs. Sungkwon 
Chung, Joonyeol Lee, and Choong-Wan Woo for valuable scientific discussion; Drs. Jae-Kyu Ryu and SoHyun Han for pulse sequence support; Chanhee Lee for MRI technical assistance; Seokwon Lee for administrative assistance.

Funding: P.T.T., S.K.L, and J.Y.P. acknowledge financial support by the Brain Research Program through the National Research Foundation of Korea funded by the Ministry of Science, ICT, and Future Planning, Project ID NRF-2019M3C7A1031993. K.M. and J.L. acknowledge financial support from the same Brain Research Program, Project ID NRF-2019M3C7A1031994. H.J.J. and J.K acknowledge financial support from the same Brain Research Program, Project ID NRF2019M3E5D2A01058328. P.T.T., S.K.L, and J.Y.P. also acknowledge financial support from the Institute for Basic Science (IBS-R015-D1).

Author contributions: P.T.T. and J.Y.P. established the methodology for direct imaging of neuronal activity. P.T.T. prepared the pulse sequence, designed and conducted all animal MRI experiments. H.J.J. and J.K. designed all optogenetic experiments for MRI and electrophysiology. H.J.J. conducted the electrophysiological and optogenetic recordings, performed fluorescence and confocal imaging. H.J.J and J.K. analyzed electrophysiological data. K.M., S.K.L. and J.L. carried out MRI measurements of relaxation time changes in T cells and simulation. P.T.T., H.J.J., K.M., S.P.K., S.K.L., J.L., J.K., and J.Y.P. contributed to data analysis and discussion. P.T.T., H.J.J., J.K., and K.M. prepared the figures and drafts. P.T.T. and J.Y.P. wrote the paper. J.Y.P. conceived and supervised the project. All authors revised the manuscript.

Competing interests: The authors declare no competing interests. 
Data and materials availability: The data can be provided upon reasonable request to corresponding authors. Email: jyparu@skku.edu (J.Y.P.) and jkwag@korea.ac.kr (J.K.).

Materials and Methods

Supplementary Text

Figs. S1 to S13

References (41-52) 

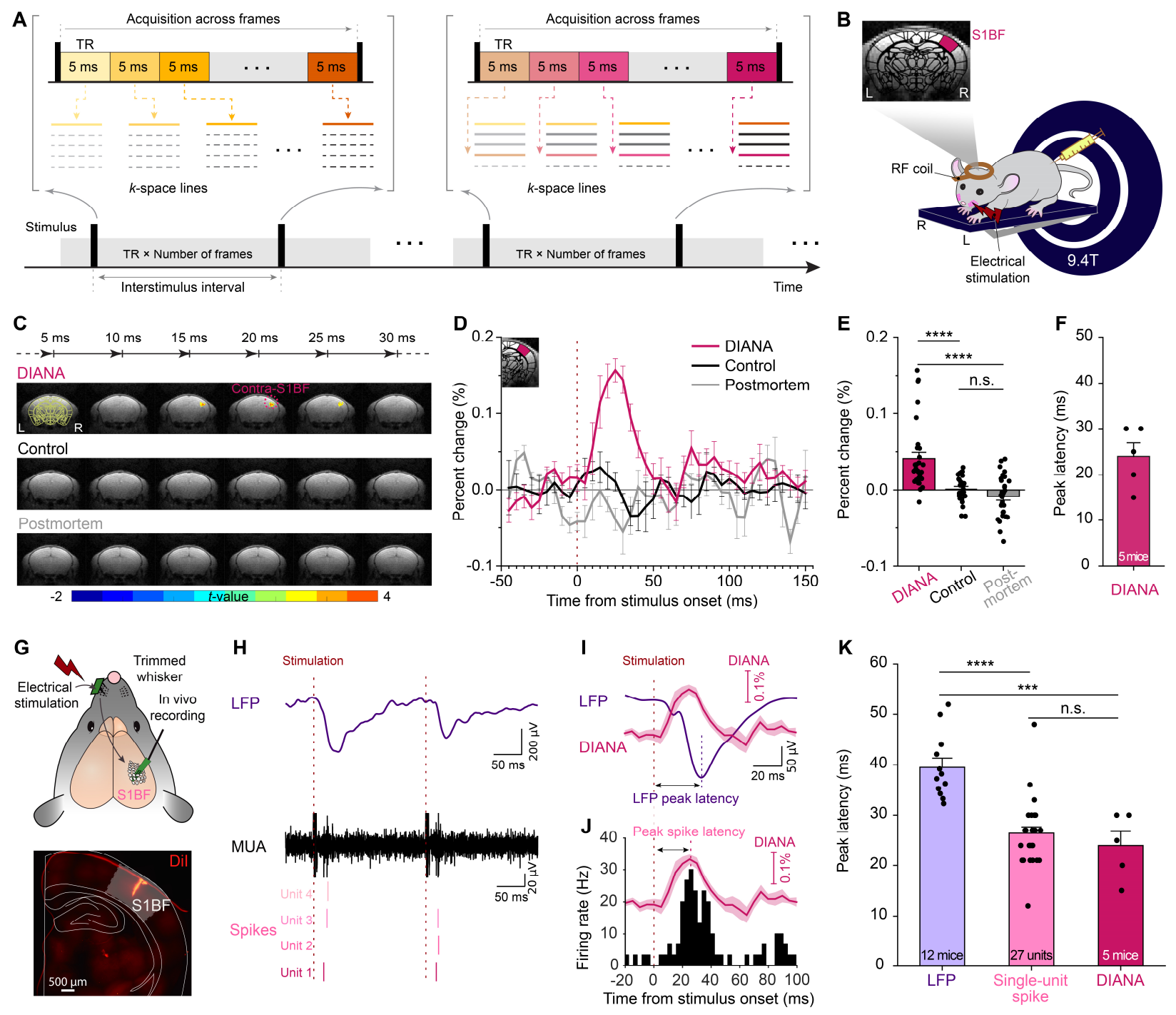

Fig. 1. DIANA-fMRI: Direct Imaging of Neuronal Activity for functional MRI with high

temporo-spatial resolution. (A) DIANA-fMRI acquisition scheme. (B) Illustration of DIANAfMRI experiment to image S1BF applying electrical stimulation to left whisker pad in an

anesthetized mouse on a $9.4 \mathrm{~T}$ scanner. ( $\mathbf{C}$ to $\mathbf{E}$ ) Time series of $t$-value maps of DIANA-fMRI in 5 ms temporal resolution (C), percent changes in DIANA signals (D) and mean signal changes during post-stimulation (E) with whisker-pad stimulation (magenta, $n=5$ mice), without stimulation (control, black, $n=5$ mice), and in postmortem condition (gray, $n=4$ mice). (F) Latency of peak DIANA response. (G) Top: Illustration of electrophysiological recording in mice 
in vivo with a 32-channel silicon probe implanted in the contralateral S1BF applying electrical whisker-pad stimulation, Bottom: Electrode track marking using a fluorescent lipophilic dye (DiI). (H) A representative local field potential (LFP) signal (purple trace, top) and multi-unit activity (MUA) (black trace, middle) from which single-unit spikes (bottom) were analyzed. Spikes of each single unit are displayed in different shades of magenta. (I and $\mathbf{J}$ ) LFP (I) and post-stimulus time histogram (PSTH) of the whisker-pad stimulation-responsive single units over time in the contralateral S1BF (J) with DIANA signals superimposed (magenta trace) for comparison. (K) Bar graph showing the latencies of LFP (purple, $n=12$ mice), the peak spike firing rate of whiskerpad stimulation-responsive single units (light magenta, $n=27$ units from 10 mice), and the DIANA response (magenta, $n=5$ mice). Vertical dotted lines indicate the whisker-pad stimulation onset time (D, and H to J) and latency of either peak LFP (I) or peak spike firing rate $(\mathrm{J})$. All data are mean \pm SEM. $* * *: p<0.001, * * * *: p<0.0001$, n.s.: $p>0.05$ for one-way ANOVA with Bonferroni post hoc test. 
A

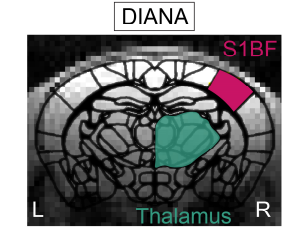

(Ipsilateral) Bregma $-1.755 \mathrm{~mm}$

(Contralateral)

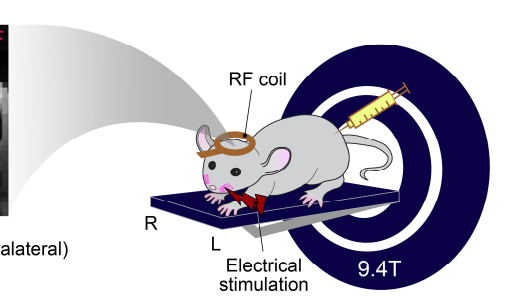

B
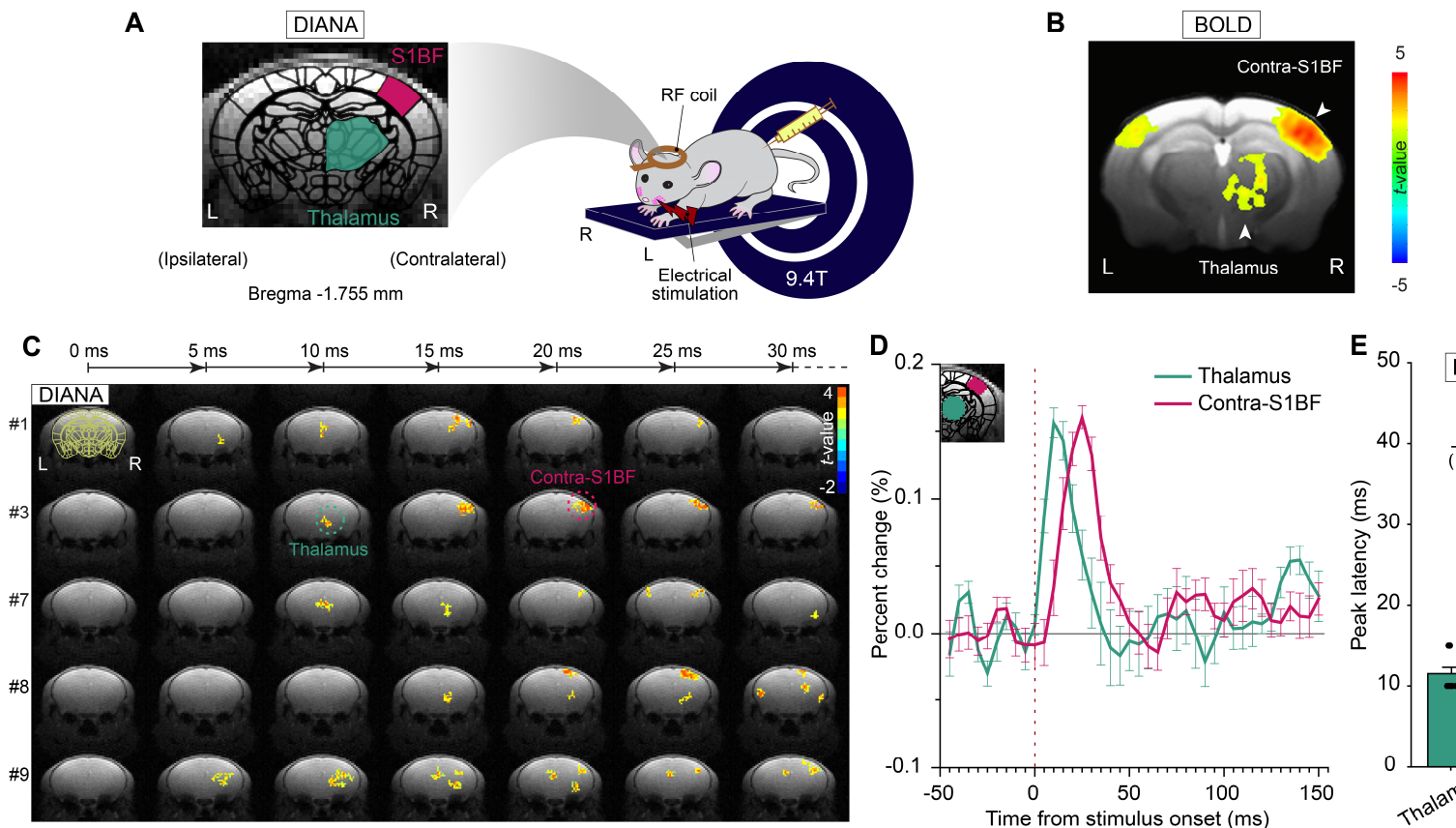

$\mathbf{D}_{0.2}$
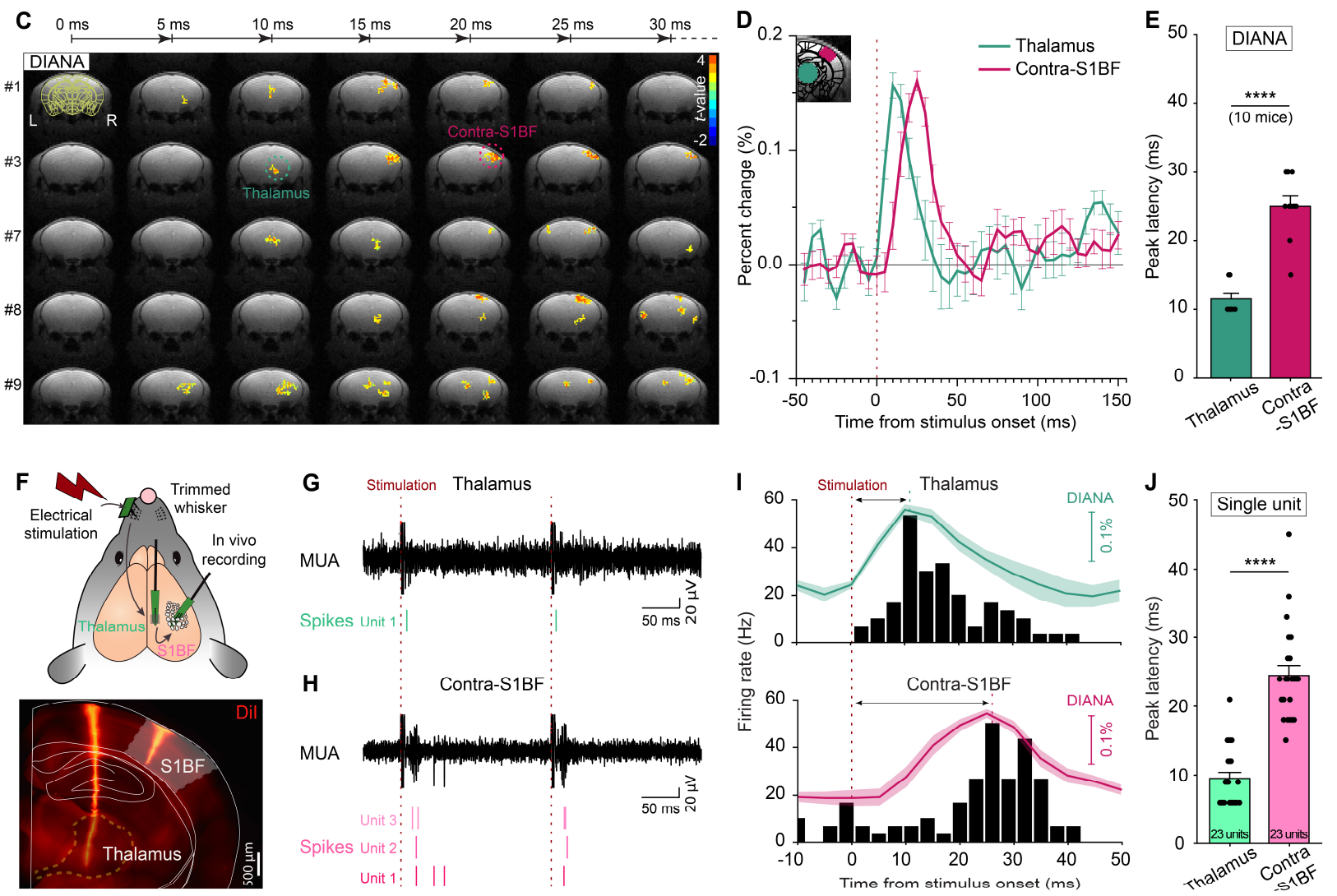

Fig. 2. High temporo-spatial resolution of DIANA-fMRI captures thalamocortical spike

propagation. (A) Illustration of DIANA-fMRI experiment to image contralateral S1BF and thalamus applying electrical stimulation to left whisker pad in an anesthetized mouse on a $9.4 \mathrm{~T}$ scanner (right) and brain imaging of a coronal slice containing both thalamus and S1BF regions (left). (B) BOLD activation map obtained as a reference $(n=10$ mice). $(\mathbf{C}$ to $\mathbf{E})$ Time series of $t$ value maps of DIANA-fMRI for $30 \mathrm{~ms}$ after whisker-pad stimulation in $5 \mathrm{~ms}$ temporal resolution from 5 representative mice (C), percent changes in DIANA signals (D), and bar graph showing the mean latencies of peak DIANA responses from thalamus (green) and contralateral S1BF 
(magenta) (E) $(n=10$ mice, $* * * *: p<0.0001$, paired $t$-test). (F) Top: Illustration of electrophysiological recording in mice in vivo with silicon probes implanted in the thalamus and the contralateral S1BF applying electrical whisker-pad stimulation, Bottom: Electrode track marking using a fluorescent lipophilic dye (DiI). ( $\mathbf{G}$ and $\mathbf{H})$ A representative multi-unit activity (MUA) (black trace, top) from which single-unit spikes (bottom) were analyzed in the thalamus (green) $(\mathrm{G})$ and the contralateral S1BF (magenta) (H). (I) Post-stimulus time histogram (PSTH) of the whisker-pad stimulation-responsive single units over time in the thalamus (top) and contralateral S1BF (bottom) with DIANA signals superimposed for comparison. (J) Bar graph showing the latencies of peak spike firing rates of whisker-pad stimulation-responsive single units recorded from the thalamus (light green, $n=23$ units from 10 mice) and contralateral S1BF (light magenta, $n=23$ units from 5 mice). Vertical dotted lines indicate the whisker-pad stimulation onset time (red) (D, and G to I) and latency of peak spike firing rate (thalamus, green; contralateral S1BF, magenta) (I). (****: $p<0.0001$, unpaired $t$-test). All data are mean \pm SEM. 
A

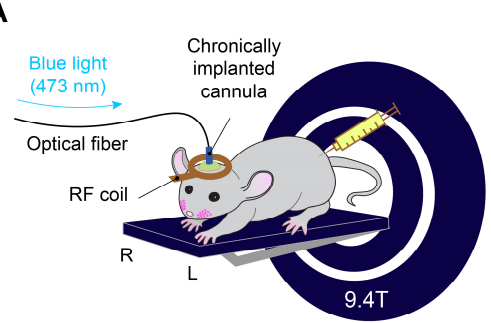

c

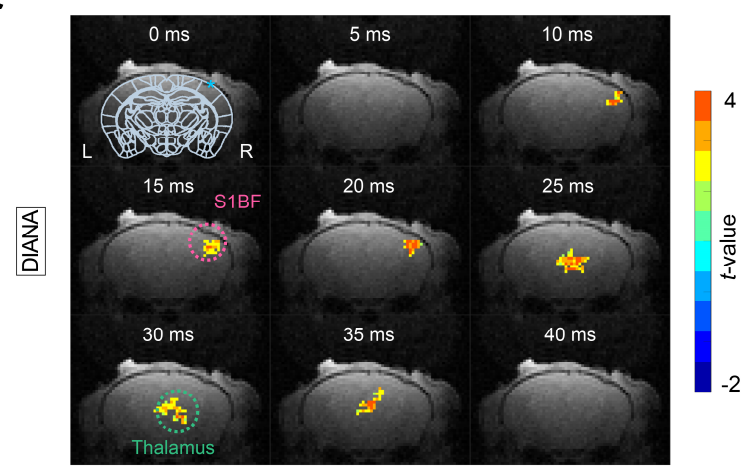

F
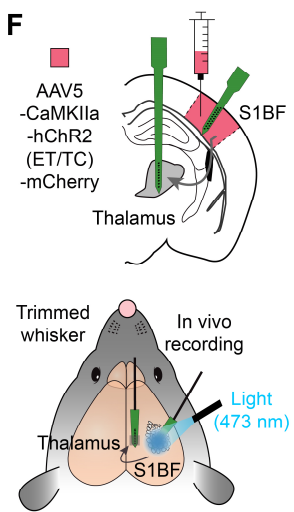

B
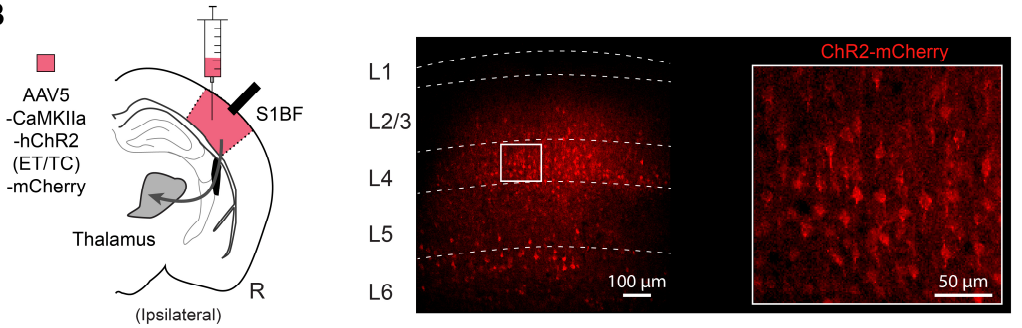

D 0
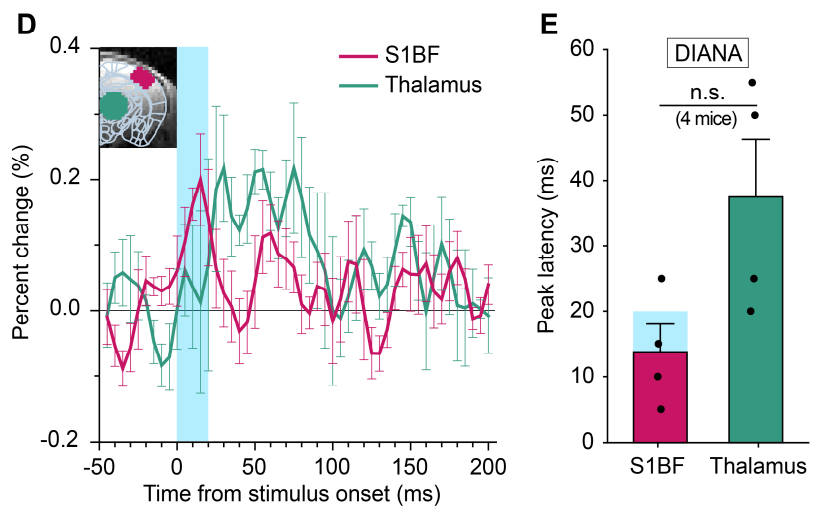

Fig. 3. Optogenetic DIANA-fMRI: DIANA responses directly detect optogenetic stimulation-

evoked spikes. (A) Illustration of optogenetic DIANA-fMRI where a fiber-optic cannula is implanted in the mouse S1BF for blue light (473 nm) stimulation. (B) Illustration of injection of AAV5-CaMKIIa-hChR2(ET/TC)-mCherry in S1BF (left) which expressed Channelrhodopsin (ChR2) to excitatory neurons across all layers of S1BF as confirmed by confocal imaging of mCherry-expressing excitatory neurons (right). (C) Time series of $t$-value maps of DIANA-fMRI from the $\mathrm{S} 1 \mathrm{BF}$ and thalamus of a representative mouse (cluster size $>5$ voxels) imaged for $40 \mathrm{~ms}$ after blue light (473 nm) stimulation onset (intensity, $50 \mathrm{~mW} / \mathrm{mm}^{2}$; duration, $20 \mathrm{~ms}$ ). (D and E) 
Percent changes in DIANA signals (D) and bar graph showing the mean latencies of peak DIANA responses in the contralateral S1BF (magenta) and thalamus (green) (E) ( $n=4$ mice, n.s.: $p>0.05$, paired $t$-test). Blue shading indicates the period of blue light stimulation. (F) Illustration of simultaneous electrophysiological recordings in vivo in the thalamus and S1BF in mice injected with AAV5-CaMKIIa-hChR2(ET/TC)-mCherry in S1BF, using the same blue light stimulation as in optogenetic DIANA-fMRI. ( $\mathbf{G}$ and $\mathbf{H}$ ) A representative multi-unit activity (MUA) (black trace, top) from which single-unit spikes (bottom) were analyzed in the contralateral S1BF (G) and the thalamus (H). (I) Post-stimulus time histogram (PSTH) of the blue light stimulation-responsive single units over time in the S1BF (top) and thalamus (bottom) with DIANA signals superimposed for comparison. (J) Bar graph showing the latencies of peak spike firing rates following the blue light stimulation in the S1BF ( $n=34$ units from 8 mice) and thalamus ( $n=7$ units from 4 mice) $(* *: p<0.01$, unpaired $t$-test). Blue shading/dotted lines indicate the period of optogenetic stimulation in S1BF (D, E, and G to J). All data are mean \pm SEM. 
A

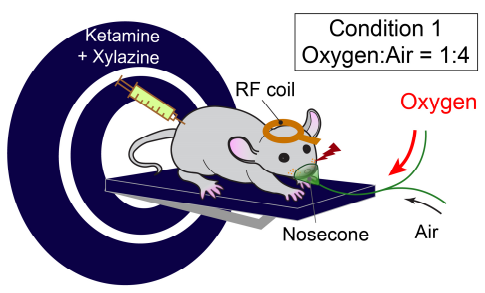

B

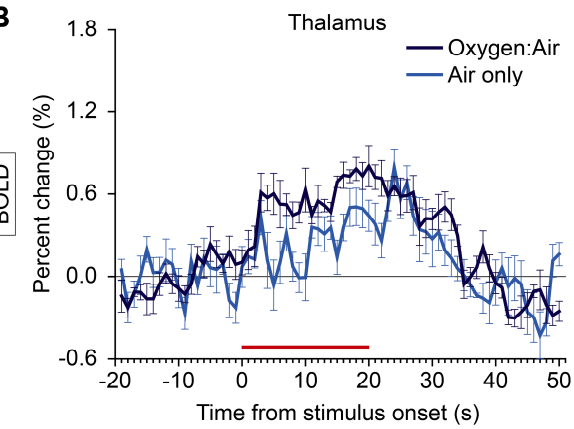

D

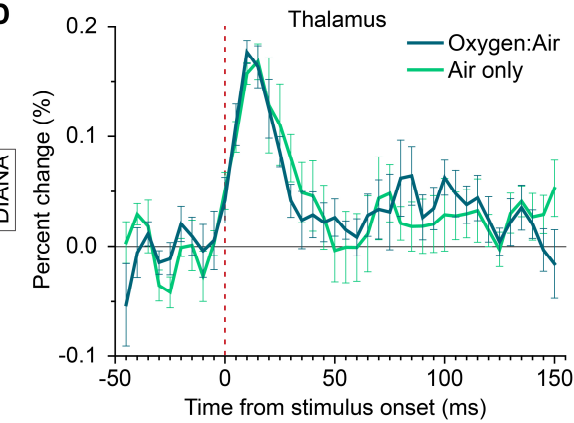

$\mathbf{F}$

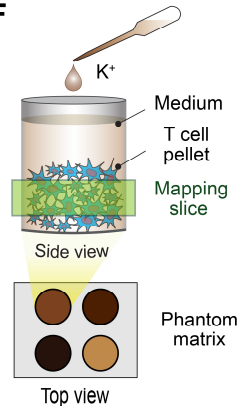

G $\quad T_{1}$ map

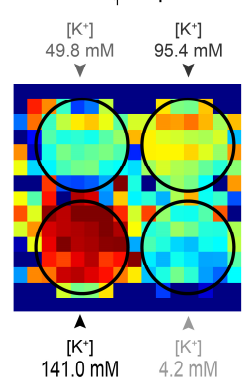

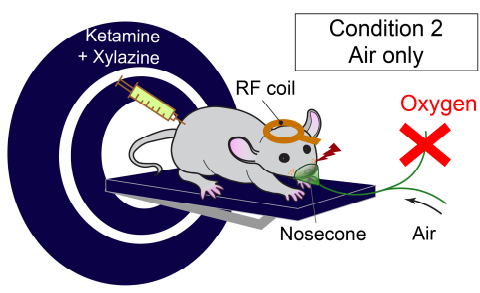
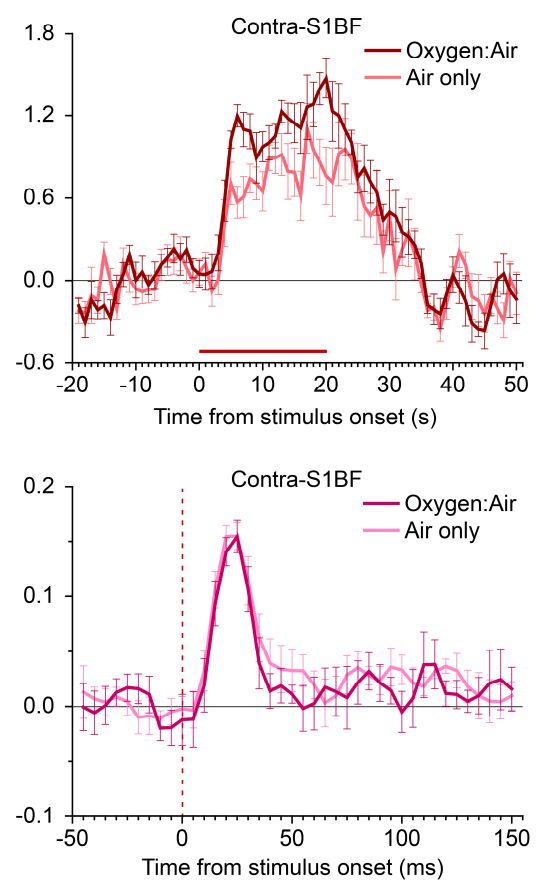
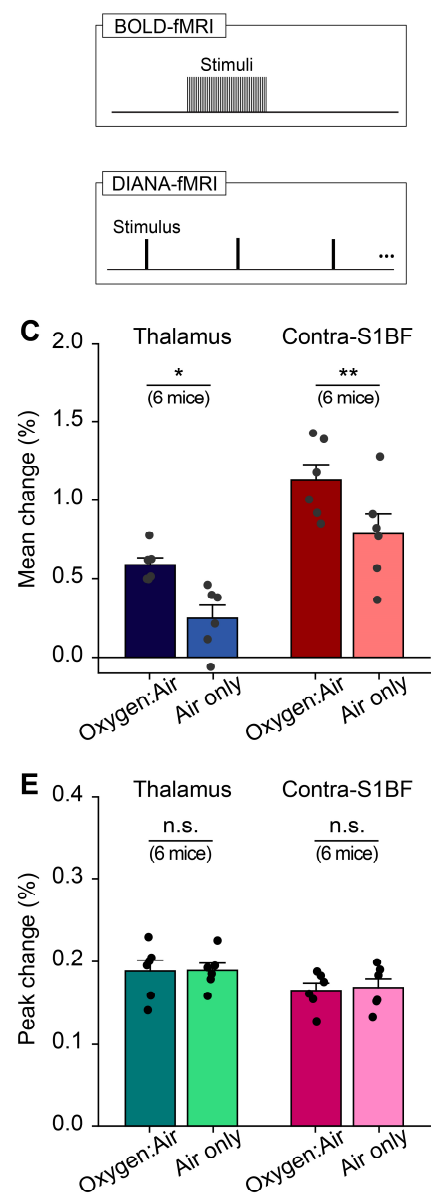
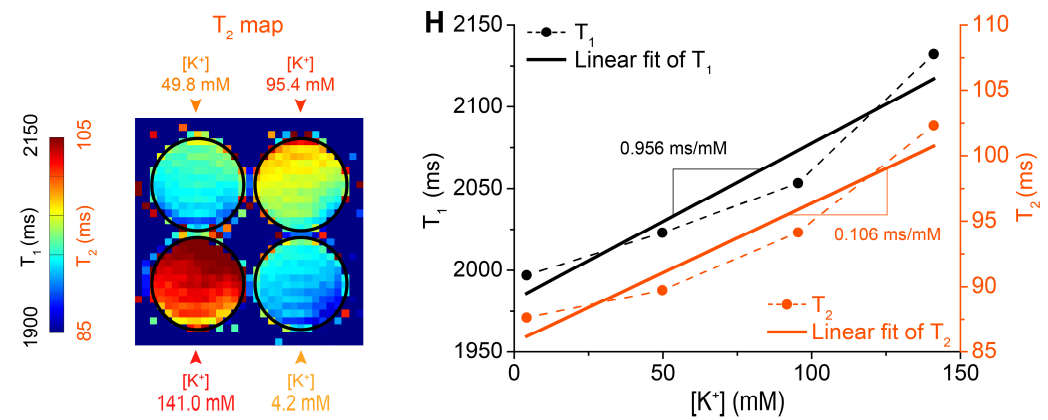

Fig. 4. BOLD-independent DIANA response and its hypothesized contrast mechanism. (A)

Illustration of oxygen challenge BOLD- and DIANA-fMRI experiments to image contralateral S1BF and thalamus applying electrical stimulation to left whisker pad in an anesthetized mouse

on a 9.4 T scanner under two conditions: One condition as a default with a mixture of extra oxygen and air (1:4) (left) and the other condition with air only (middle) at the same flow rate. The same whisker-pad electrical stimulus (strength, $0.5 \mathrm{~mA}$; pulse duration, $0.5 \mathrm{~ms}$ ) was used for both 
BOLD- and DIANA-fMRI stimulation paradigms (right). (B and C) Percent signal changes of BOLD responses obtained from the thalamus $(\mathrm{B}, \mathrm{left})$ and the contralateral $\mathrm{S} 1 \mathrm{BF}(\mathrm{B}$, right) under two conditions, and the corresponding bar graph showing the mean signal changes of BOLD responses in the thalamus and contralateral $\mathrm{S} 1 \mathrm{BF}(\mathrm{C}, n=6$ mice $)$. The horizontal red bar indicates the period of electrical stimulation in BOLD-fMRI. (D and E) Same as (B and C) but with responses acquired using DIANA-fMRI ( $n=6$ mice). Vertical dotted lines indicate the stimulation onset time. (F) Illustration of a phantom experiment using $\mathrm{T}$ cells. (G) $\mathrm{T}_{1}$ and $\mathrm{T}_{2}$ relaxation times maps acquired at different extracellular $\mathrm{K}^{+}$concentrations $\left(\left[\mathrm{K}^{+}\right]\right)$ranging from 4.2 to $141.0 \mathrm{mM}$. (H) Plots of $\mathrm{T}_{1}$ (black dotted line) and $\mathrm{T}_{2}$ (orange dotted line) changes with respect to $\left[\mathrm{K}^{+}\right]$. Solid lines are the linear fits to the $T_{1}$ and $T_{2}$ values to calculate the correlation coefficients with respect to $\left[\mathrm{K}^{+}\right]$. All data are mean \pm SEM. $*: p<0.05, * *: p<0.01$, n.s.: $p>0.05$ for paired $t$-test. 